\section{Response to Comment on "Declining Wild Salmon Populations in Relation to Parasites from Farm Salmon"}

\author{
Martin Krkošek, ${ }^{1,{ }^{2 *}} \dagger$ Jennifer S. Ford, ${ }^{3}$ Alexandra Morton, ${ }^{4}$ Subhash Lele, ${ }^{1}$ Mark A. Lewis ${ }^{1,2}$ \\ We evaluated the effect of sea lice (Lepeophtheirus salmonis) infestations on wild pink \\ salmon (Oncorhynchus gorbuscha) populations in the Broughton Archipelago, British Columbia. \\ Riddell et al. suggest that we ignored factors and selectively used data. Here, we clarify \\ misunderstandings and provide analysis to test the strength of our conclusions.
}

$\mathrm{W}$ e agree with Riddell et al. (1) that many factors affect salmon survival. In our study of the effects of sea lice, we controlled for nonlouse factors, using the stochastic Ricker model in a comparative analysis (2). The model accounts for density-dependent mortality and environmental variation in survival (3), two mortality factors not associated with sea lice. We applied the model comparatively to exposed pre-infestation, exposed infested, and unexposed populations. The exposed pre-infestation and unexposed populations share many factors affecting pink salmon population dynamics, evidenced by similar population growth rates (2) and synchronous population dynamics (4). The main difference between unexposed populations and exposed populations is that the former did not experience infestations $(5,6)$, whereas the latter experienced a series of infestations $(5,7,8)$. Because the exposed infested populations have a depressed population growth rate $(r)$, this indicates that sea lice infestations have driven the difference in $r$ between exposed and unexposed populations. Further inclusion of sea lice abundance estimates improved the model fit and showed that increased louse abundance is associated with lower survival (2).

The spatial time series data we analyzed have temporal and spatial correlation, raising the issue of pseudoreplication and its impact on statistical inference. We controlled for temporal correlation, using parametric bootstrapping of the stochastic Ricker model to generate confidence intervals for $r$ (3). Spatial correlation is known for salmon populations but occurs at a large scale that encompasses both exposed and unexposed populations, indicating synchronous regional environmental variation (4). An appropriate statistical analysis in this situation follows a matched case-control

${ }^{1}$ Centre for Mathematical Biology, Department of Mathematical and Statistical Sciences, University of Alberta, Edmonton, AB, Canada. '2Department of Biological Sciences, University of Alberta, Edmonton, AB, Canada. ${ }^{3}$ Ecology Action Centre, Halifax, NS, Canada. ${ }^{4}$ Salmon Coast Field Station, Simoom Sound, BC, Canada.

*To whom correspondence should be addressed. E-mail: mkrkosek@ualberta.ca

†Present address: School of Aquatic and Fishery Sciences, University of Washington, Seattle, WA 98105, USA. design in epidemiology (9), where subjects experiencing the same environmental variation are divided into a treatment group (exposed populations) and control group (unexposed populations). After conditioning on the shared common environmental variation, the spatial replicates can be considered independent observations on differences in population growth rate between exposed and unexposed populations. The crucial result is that growth rate $r$ is similar for control and treatment groups before infestations and then declines significantly for the exposed group during infestations but not the unexposed group (Table 1). A potential flaw in this analysis is that the assignment to control and treatment groups was not random but rather was based on proximity to salmon farms and sea lice infestation. Although the assumption of common environmental variation is well supported, it is not impossible that a nonlouse factor changed systematically in a negative way for exposed populations but not for unexposed populations during infestation years. However, neither Riddell et al. (1) nor we have been able to identify such a confounding factor. Furthermore, the estimated effect of sea lice is consistent with predictions from other independent analyses that established and quantified the underlying mechanisms of transmission and mortality $(8,10)$.

Riddell et al. suggest that it was inappropriate to exclude the 2004 data from our analysis. These data were not excluded but rather were assigned to a separate "fallow" category in accordance with the management intervention. In spring 2003, the provincial and federal governments implemented the Pink Salmon Action Plan, which involved fallowing the primary migration route we identified in (2). This constitutes a major management intervention that had not been conducted before or replicated since. The fallowing management action was associated with a significant reduction in L. salmonis abundance in 2003 (11) and high marine survival for that cohort (12) (Table 1). Contrary to Riddell et al.'s interpretation, our analysis estimated the survival for pink salmon over their entire life cycle, not just the early out-migration period. Further fallowing interventions may help restore pink salmon, but the fallow treatment needs replication before its effects can be robustly assessed.

Riddell et al. (1) argue that the beginning of the infestation period (2001 out-migration and 2002 return) is confounded by high spawner abundance in 2000. We agree that density-dependent mortality likely contributed to the 2002 collapse, and we controlled for this in our analysis by using a density-dependent population growth model. The Ricker model shows that pink salmon are commonly in the overcompensation range where high spawner abundance leads to low returns (Fig. 1). Even in the absence of sea louse infestation, the Ricker curve predicts a drop from an average of 2.6 times historical abundance in 2000 to a mean of 0.78 times historical abundance in 2002, a decline by a factor of 3.3. The observed mean abundance in 2002 was 0.085 times historical abundance, which is a decline by a factor of 31, indicating that a density-independent factor was the primary cause of the collapse. Riddell et al. (1) are incorrect that our results depend on setting 2000 as the start of the infestation period. Annual survival during all the infestation years was mostly negative (Table 2), and excluding the 2000 to 2002 collapses from the data yields an estimated five generations to reach $99 \%$ loss $(r=-0.85$; 95\% CI: -1.48 to -0.25 for exposed populations during infestation years), as opposed to four generations, as estimated in our original report (2).

Riddell et al. (1) are concerned that excluding the Glendale but including the Kakweiken in the analysis may affect the results. This criticism has already been addressed and shown to have no effect on the results (13). Following the method in (2), with Glendale included, the population

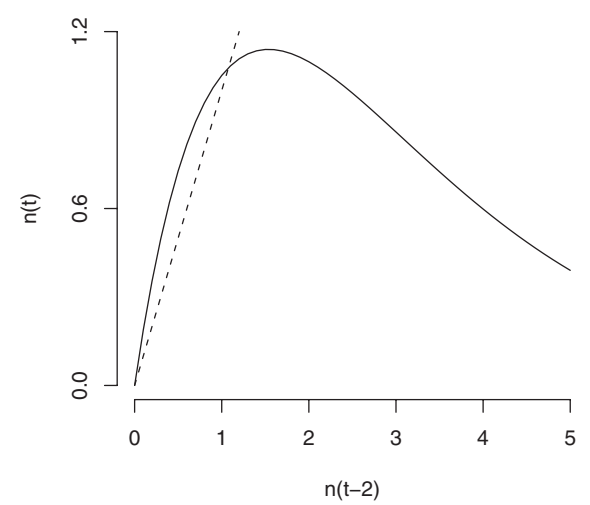

Fig. 1. Plot of the Ricker map $n(t)=n(t-2) \exp$ $[r-b n(t-2)]$ parameterized by pre-infestation (1970 to 2001) escapement data for Broughton Archipelago pink salmon $[n(t)$ is an escapement estimate in year $t$ normalized by its time-series mean (2)]. The dotted line is the 1:1 line showing the carrying capacity at the intersection with the Ricker curve. Overcompensation occurs where the curve has negative slope, indicating that high spawner abundance leads to low returns. Comparison with figure 3 in (2) shows that pink salmon were commonly in the overcompensatory regime, including the exposed infested group. 
Table 1. Point estimates and $95 \%$ confidence intervals for the population growth rate, $r$, of pink salmon for exposed and unexposed populations before and during the sea lice infestations and the fallow. Small deviation in estimates from those in the corrected results $(16,17)$ are due to dividing the unexposed populations into Before Infestations, During Infestations, and Fallow groups in the model fits to get the point estimates of the parameters and also due to stochasticity in the bootstrap simulations to get the confidence intervals.

\begin{tabular}{lccr} 
& Before infestations & During infestations & Fallow \\
\hline Exposed & $0.70(0.49,0.91)$ & $-1.16(-1.66,-0.60)$ & $2.63(1.39,3.79)$ \\
Unexposed & $0.63(0.55,0.70)$ & $0.78(0.50,1.08)$ & $-0.05(-0.59,0.62)$ \\
\hline
\end{tabular}

Table 2. Annual survival estimates, $\ln [n(t) / n(t-2)]$ per pink salmon population in the Broughton Archipelago during 2002 to 2006.

\begin{tabular}{llrrrr} 
River & 2002 & 2003 & $2004^{*}$ & 2005 & 2006 \\
\hline Ahta & -4.48 & -3.16 & 3.40 & 1.05 & -2.73 \\
Kakweiken & -5.03 & -1.93 & 2.46 & 0.75 & -0.55 \\
Kingcome & -3.79 & -1.08 & 2.63 & -1.94 & -1.93 \\
Wakeman & -2.54 & 1.02 & 0.86 & -3.65 & -1.55 \\
Viner & -0.51 & -2.10 & 1.57 & -1.05 & -3.02 \\
Lull & -6.73 & -5.31 & 4.28 & 3.55 & -3.16 \\
Ahnuhati & -4.11 & 0.40 & 2.82 & -0.01 & -2.54 \\
Glendale & -3.73 & -2.12 & 3.59 & 0.32 & -1.29 \\
Average & -3.87 & -1.79 & 2.70 & -0.12 & -2.10 \\
\hline
\end{tabular}

*This year corresponds to the pink salmon cohort whose juvenile out-migration occurred during the fallow year 2003.

growth rate for Broughton pink salmon populations during the sea lice infestations is $-1.00(95 \%$ CI: -1.52 to -0.52 ), and without Glendale and Kakweiken, the population growth rate is -1.23 (95\% CI: -1.80 to -0.62 ). The population growth rate for Broughton pink salmon populations during the infestations as reported in our paper is $r=$ -1.17 (95\% CI: -1.71 to -0.59$)$.

Riddell et al. (1) also suggest that competition with fish from the Glendale spawning channel contributed to declines in other Broughton pink salmon populations. Unfortunately, they provide no quantitative support for this hypothesis and fail to explain why it is apparent in only half the data (odd but not even years in their figure 1) and only in pre-infestation years. Figure 2 in (2) shows that the marked decline in pink salmon during the infestations is unprecedented. Figure 1 in Riddell et al. (1) indicates the Glendale populations fluctuated synchronously with the other populations in response to the infestations. If their hypothesis were correct, the Glendale populations would have fluctuated opposite to - not synchronously with - the other populations. The common factors that explain recent differences in the fluctuations of pink salmon abundance in the Broughton Archipelago relative to unexposed populations are the sea lice infestations and the fallow treatment.
Riddell et al. supply encouraging news that 2007 escapement estimates indicate improved survival. This is only inconsistent with our analysis if 2006 infestations remained unchanged from recent nonfallow years, but Riddell et al. provide no supporting data. The impacts of salmon aquaculture on sympatric wild salmon stocks are now known to be widespread (14), and solutions are possible given sufficient political will (15). Our analysis indicates a critical sea lice threshold of $r^{*} / \alpha=1.3$ motile lice per juvenile pink salmon, below which population declines can be reversed (2). We agree with Riddell et al. that management needs to consider many factors affecting wild salmon. Prevention of sea lice infestations of wild juvenile salmon is a management and policy option that may help restore wild salmon.

\section{References and Notes}

1. B. E. Riddell, R. J. Beamish, L. J. Richards, J. R. Candy, Science 322, 1790 (2008); www.sciencemag.org/cgi/ content/full/322/5909/1790b.

2. M. Krkošek et al., Science 318, 1772 (2007).

3. B. Dennis, M. L. Taper, Ecol. Monogr. 64, 205 (1994).

4. B. J. Pyper, F. J. Mueter, R. M. Peterman, D. J. Blackbourn, C. C. Wood, Can. J. Fish. Aquat. Sci. 58, 1501 (2001).

5. A. Morton, R. Routledge, C. Peet, A. Ladwig, Can. J. Fish. Aquat. Sci. 61, 147 (2004)

6. C. R. Peet, thesis, University of Victoria (2007).

7. A. B. Morton, R. Williams, Can. Field Nat. 117, 634 (2003).

8. M. Krkošek, M. A. Lewis, A. Morton, L. N. Frazer, J. P. Volpe, Proc. Natl. Acad. Sci. U.S.A. 103, 15506 (2006).

9. K. J. Rothman, Modern Epidemiology (Little Brown, Boston, 1986).

10. M. Krkošek et al., Proc. R. Soc. London B Biol. Sci. 274, 3141 (2007)

11. A. Morton, R. D. Routledge, R. Williams, N. Am. J. Fish. Manage. 25, 811 (2005).

12. R. J. Beamish et al., ICES J. Mar. Sci. 63, 1326 (2006).

13. M. Krkošek et al., Rev. Fish. Sci. 16, 413 (2008).

14. J. S. Ford, R. A. Myers, PLoS Biol. 6, e33 (2008).

15. A. A. Rosenberg, Nature 451, 23 (2008).

16. Corrections and Clarifications, Reports, Krkošek et al., Science 322, 1790 (2008)

17. See revised supporting online material for (2) at www. sciencemag.org/cgi/content/full/318/5857/1772/DC1.

18. Funding came from the Natural Science and Engineering Research Council of Canada, the Canadian Mathematics of Information Technology and Complex Systems National Centre of Excellence Network on Biological Invasions and Dispersal Research (with nonacademic participants including the David Suzuki Foundation, Canadian Sablefish Association, Wilderness Tourism Association, Watershed Watch Salmon Society, and Finest at Sea), the National Geographic Society, Tides Canada, a University of Alberta Bill Shostak Wildlife Award, the Lenfest Ocean Program, Census of Marine Life, and a Canada Research Chair.

10 March 2008; accepted 16 September 2008 10.1126/science. 1156578 suggested.

Referring to extirpation rather than local extinction implies that recovery is possible. This is not the case under a regime of infestations and negative population growth rates; extant populations will be lost and recovery efforts will fail. ulations be lost, colonization must come from outside the Broughton rather than populations within it; recovery would be more greatly com- 